\title{
CT System Parameter Calibration and Imaging Issues
}

\author{
Jie Peng \\ North China Electric Power University Baoding 07100, China \\ pengjie@ncepu.cn
}

Keywords: The basic principle of CT imaging; Radon transform; Attenuation factor; Rotation center

Abstract: The article takes the A model of the National University Student Modeling Contest in 2017 as an example to study CT system parameter calibration and imaging issues. From the questions we can see, the topic is to calibrate the parameters of the CT system according to the sample (template) of known structure and obtain the related CT scan information (position, geometry, absorption rate) of other unknown objects.

\section{Problem Analysis}

Analysis of the data in Appendix A of Question A shows that the calibration template is divided into 256*256 pixel units. The absorption intensity of each pixel unit is given in the table of Annex I. The absorption intensity of the region where the uniform solid medium is located is 1 , and other regions Absorption intensity is 0 . Therefore, the data is binarized, and the pixel units having different absorption intensities are marked. As can be seen from the drawing, the position of the pixel unit where the data is 1 constitutes an ellipse and a circle. The two-dimensional graphics of the media.

When a parallel x-ray beam hits a two-dimensional test medium, it passes through different sets of pixel units. Because the length of the medium to be measured is different in the direction of the ray, the energy of the ray after the x-ray passes through the medium is also different. Therefore, the strength of the signal received by the receiver is also different. By analyzing the data in Annex II, according to different angles, the received signal strength of the receiver is different, Radon transform and inverse transform are used to determine the position of the center of rotation.

First of all, the data in Annex II is binarized. In the upper right corner of the figure, the arc part is the absorption of X-rays at different angles of the ball. The width of the arc reflects the diameter of the ball, so the ratio can be The total length of the detector cells is obtained, from which the distance between the cells is found to be $0.2857 \mathrm{~mm}$.

\section{Model establishment and solution}

Calibrate the CT system based on the relevant information of the calibration template of the known information. According to the questions on the first question, we use the radar transform to calculate. Distance light that is a unit pixel passes through the image, then the radon transform calculates the integral through the image length as: 


$$
R_{\theta}\left(x^{\prime}\right)=\int_{n}^{+\infty} f\left(x^{\prime} \bullet \cos \theta-y^{\prime} \bullet \sin \theta, x^{\prime} \bullet \sin \theta+y^{\prime} \cos \theta\right) \mathrm{d} y
$$

The line integral of $f(x, y)$ in the vertical direction is $f(X, Y)$ projected onto the $X$ axis; the integration in the horizontal direction is $f(X, Y)$ projected onto the $Y$ axis. The projection can be calculated at any angle $\theta$. The figure below illustrates the geometry of the Radon transform along the angle $\theta$.

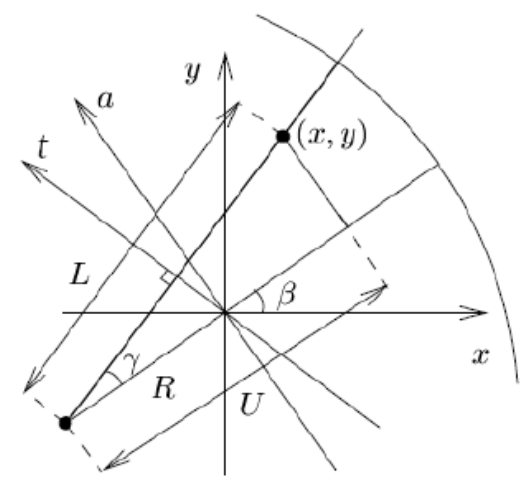

Figure 1. Parallel beam projection geometry

The parameters in Fig.1 are explained as follows:

1) $\mathrm{XOY}$ is a Cartesian coordinate system with the origin at point $\mathrm{O}$.

2) $\mathrm{XrOYr}$ is a rotating coordinate system with the origin at point $\mathrm{O}$.

The angle between the $\mathrm{Xr}$ axis and the $\mathrm{X}$ axis is $\Phi, \gamma, \theta$ are polar coordinates, and the angle between the radial direction $\mathrm{r}$ and the $\mathrm{x}$ axis is $\theta$.

Let the image to be built be $\mathrm{a}(\mathrm{x}, \mathrm{y})$, and its two-dimensional Fourier transform is $\mathrm{A}\left({ }^{\omega_{1}}, \omega_{2}\right)$, then:

$$
\mathrm{a}(x, y)=\frac{1}{4 \pi^{2}} \int_{-\infty-\infty}^{+\infty+\infty} \int_{-\infty} A\left(\omega_{1}, \omega_{2}\right) e^{j\left(x \omega_{1}+y \omega_{2}\right)} d \omega_{1} d \omega
$$

Convert each physical quantity in (4) to the quantity expressed in polar coordinates

$$
\begin{gathered}
\left(\omega_{1}, \omega_{2}\right) \longrightarrow P(f, \phi) \\
x \omega_{1}+y \omega_{2}=2 x \pi f \cos \phi+2 y \pi f \sin \phi=2 \pi f \cos (\theta-\phi)
\end{gathered}
$$

To convert the expressions of $\mathrm{d} \omega_{1}$ and $\mathrm{d} \omega_{2}$ to the expression represented by $d f$ and $\mathrm{d} \phi$, it is necessary to introduce the Jacobian determinant and then convert it, that is:

$$
\mathrm{a}(x, y)=\frac{1}{4 \pi^{2}} \int_{-\infty}^{+\infty} A\left(\omega_{1}, \omega_{2}\right) e^{j\left(x \omega_{1}+y \omega_{2}\right)} d \omega_{1} d \omega_{2}
$$

Note the physical meaning of Eq.2 (3.9), look at the second integral:

$$
|f| P(f, \phi) e^{j 2 \pi f y \cos (\theta-\phi)} d f
$$

This can be written as the inverse Fourier transform of the spatial domain variable $X_{\mathrm{r}}$ : 


$$
\int_{-\infty}^{+\infty}|f| P(f, \phi) e^{j 2 \pi f \gamma \cos (\theta-\varphi)} d f=h\left(x_{r}\right) * P\left(x_{r}, \phi\right)=g\left(x_{r}, \phi\right)
$$

$\mathrm{H}(\mathrm{Xr})$ and $\mathrm{P}(\mathrm{Xr}, \Phi)$ are Fu Liye transforms of $|\mathrm{f}|$ and $\mathrm{P}(\mathrm{F}, \Phi)$. The physical meaning of Eq.7 is exactly the value of the modified projection $\mathrm{g}(\mathrm{Xr}, \Phi)$ obtained by filtering the projection $\mathrm{P}(\mathrm{Xr}, \Phi)$ through a filter whose transfer function is $|\mathrm{f}|$ in the frequency domain when $\mathrm{Xr}=\mathrm{y} \cos (\theta, \Phi)$ is satisfied. The $\mathrm{Xr}=\mathrm{y} \cos (\theta, \Phi)$ is exactly the ray equation given to the fixed point $(\mathrm{y}, \theta)$.

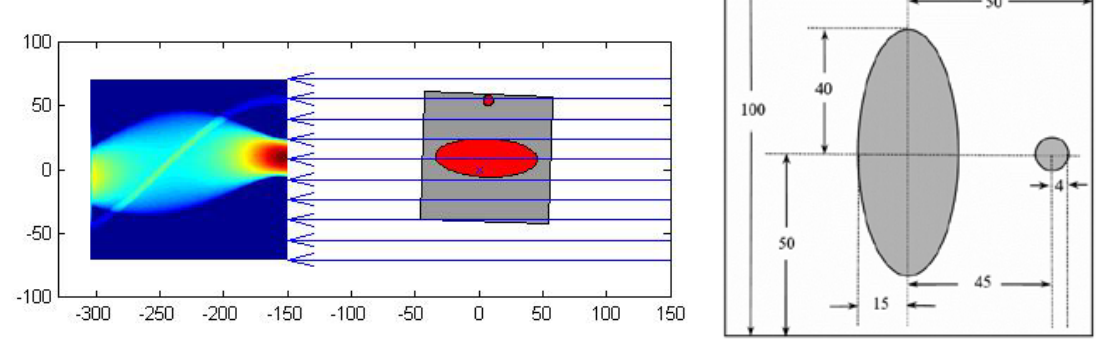

Figure 2. Schematic diagram of parameter calibration

The data in Annex 2 are plotted using MATLAB to obtain the absorption intensity image as shown in Fig. 2and compared with the template schematic diagram provided. In the absorption intensity image, the coordinates of the $\mathrm{X}$ axis represent the rotation angle, and the coordinates of the $\mathrm{Y}$ axis represent the vertical distance of the sensor. The longer the ray passes through the object, the greater the energy absorbed by the object. At the same time, it also represents the greater absorption intensity. The data is processed by matlab, and the point $\mathrm{A}$ of the maximum data is obtained. The coordinates of the point A are 151, 223. Similarly, when projected from the short axis of the ellipse, the data is highly symmetrical, and its data coordinate is $\mathrm{B}(61,235)$, and the width of the object is LA1 = 73-46 = 27, LA2 = 277-169 = 108, LB = 307 for two scans.

Observed data in two directions have been obtained. The direction of the line connecting the two circle centers to the right is the positive direction of the $\mathrm{X}$ axis, the direction of the long axis of the ellipse is the positive direction of the $\mathrm{Y}$ axis, and the center of the ellipse is the origin.

The angle of each rotation is1 degrees.The distance between detector elements is $\mathrm{L}=8 / 28=0.2857 \mathrm{~mm}$. The position of the rotation center of the CT system in the square pallet.

$\mathrm{X}$ direction: $\Delta \mathrm{X}=(512 / 2-223) \times \Delta \mathrm{L}=-9.1806 \mathrm{~mm}$

$\mathrm{Y}$ direction: $\Delta \mathrm{Y}=(512 / 2-235) \times \Delta \mathrm{L}=5.8422 \mathrm{~mm}$

\section{References}

[1] Hui Miao. Cone beam 3D XCT reconstruction algorithm [D]. North Central University, 2007. (In Chinese)

[2] Zhang Xuesong, Zhao Baishan.Cup artifact correction for CT images based on Radon transform[J/OL].CT theory and application, 2016,25 (05): 539-546.(In Chinese)

[3] Yanshulin, Niu Yantao. Explanation of medical imaging technical terms [J].Chinese Journal of Medical Imaging, 2010, 18 (06): 524.(In Chinese)

[4] Jiang Qiyuan, Xie Xing Jin. Ye Jun, mathematical model [M]. Beijing: Higher Education Press, 2003.8.(In Chinese)

[5] Huang Ke Chun.CT mathematical model of system parameter calibration [J]. Journal of Jiaying University, 2018,36 (08): 16-21.(In Chinese) 